\title{
The comparison of clinically relevant doses of intrathecal ropivacaine and levobupivacaine with fentanyl for labor analgesia
}

\author{
Kyung-Mi Kim ${ }^{1}$, Young Wan Kim², Ji Won Choi ${ }^{3}$, Ae Ryoung Lee ${ }^{2}$, and Duck Hwan Choi ${ }^{2}$ \\ Department of Anesthesiology and Pain Medicine, ${ }^{1}$ Hallym University Dongtan Sacred Heart Hospital, Hwaseong, ${ }^{2}$ Samsung Medical \\ Center, Sungkyunkwan University School of Medicine, Seoul, ${ }^{3}$ Daejin Medical Center, Bundang Hospital, Seongnam, Korea
}

Background: Intrathecal labor analgesia using new local anesthetics such as ropivacaine or levobupivacaine becomes more popular by virtues of their safety and decreased motor weakness. However, the analgesic efficacy of the clinically effective intrathecal doses of these new local anesthetics combined with fentanyl has yet to be determined.

Methods: Sixty parturients who requested neuraxial analgesia in early active labor were randomly assigned to either ropivacaine (group R, $\mathrm{n}=30$ ) or levobupivacaine (group $\mathrm{L}, \mathrm{n}=30$ ) group. Group $\mathrm{R}$ received $3 \mathrm{mg}$ of intrathecal ropivacaine and the group L received $3 \mathrm{mg}$ of intrathecal levobupivacaine mixed with $20 \mu \mathrm{g}$ of fentanyl as part of a combined spinalepidural (CSE) technique. The associated block parameters, such as pain scores, duration of analgesia, the highest levels of the sensory block and motor block scores 30 mins after the injection were compared between two groups.

Results: Intrathecal ropivacaine offered shorter analgesia $(87 \pm 41 \mathrm{~min}$ vs. $122 \pm 56 \mathrm{~min}, \mathrm{P}<0.05)$ with lower sensory height (T8.5 vs. T6, $\mathrm{P}<0.05$ ) and led to lower incidence of complete analgesia $(73$ vs. $97 \%, \mathrm{P}<0.05)$ compared with intrathecal levobupivacaine. Although motor weakness was comparable in both groups, significantly weak perineal squeezing was noticed in Group L ( 7 of 30 parturients vs. 16 of 30, P $<0.05$ ).

Conclusions: Clinically relevant doses of intrathecal levobupivacaine in combination with fentanyl as part of a CSE technique provides more effective analgesia than equivalent doses of intrathecal ropivacaine in early labor, but is accompanied by slight motor weakness. (Korean J Anesthesiol 2013; 65: 525-530)

Key Words: Combined spinal-epidural anesthesia, Intrathecal injection, Labor analgesia, Levobupivacaine, Ropivacaine.

Received: December 12, 2012. Revised: 1st, April 26, 2013; 2nd, May 31, 2013. Accepted: May 31, 2013.

Corresponding author: Duck Hwan Choi, M.D., Ph.D., Department of Anesthesiology and Pain Medicine, Samsung Medical Center, Sungkyunkwan University School of Medicine, 50, Ilwon-dong, Gangnam-gu, Seoul 135-710, Korea. Tel: 82-2-3410-0369, Fax: 82-2-3410-0361, E-mail: duckhwan.choi@samsung.com

(c) This is an open-access article distributed under the terms of the Creative Commons Attribution Non-Commercial License (http:// creativecommons.org/licenses/by-nc/3.0/), which permits unrestricted non-commercial use, distribution, and reproduction in any medium, provided the original work is properly cited. 


\section{Introduction}

A combined spinal-epidural (CSE) technique has become one of the best options for labor analgesia. Usually the initial intrathecal analgesia via CSE is rapidly obtained using a low-dose local anesthetic combined with a lipophilic opioid in early labor [1]. Although racemic bupivacaine has been used most extensively, the new S-enantiomer drugs such as ropivacaine and levobupivacaine, have recently become popular for intrathecal (IT) labor analgesia because they have been reported to have less cardiovascular and neurologic side effects in addition to less motor block [2-4].

When comparing the difference in potency for labor analgesia between the above two drugs or among three drugs, up-anddown sequential allocation technique has been used via epidural or IT route. This technique was aimed at finding the median effective dose (ED50), which is interpreted as the minimum local anesthetic dose (MLAD) or concentration (MLAC) [5]. Both ropivacaine and levobupivacaine when injected epidurally were less potent than racemic bupivacaine [6-8], but levobupivacaine and ropivacaine showed similar potency $[9,10]$. Among the studies comparing IT analgesic efficacy of these local anesthetics using the same method, Camorcia et al. [11] demonstrated a potency hierarchy of spinal bupivacaine $>$ levobupivacaine $>$ ropivacaine. However, these studies using MLAC or MLAD did not completely reflect the dose-response relation of these three drugs. Next, an another study using a full dose-response technique was performed to provide information about entire shape and slope of the dose-response curve, which showed that bupivacaine was most potent and ropivacaine and levobupivacaine were similar in potency [12].

Furthermore some studies using a specific clinical doses of IT ropivacaine and levobupivacaine revealed no differences in both analgesic potency and side effects between two IT local anesthetics $[4,13]$. However, IT local anesthetics was used as the sole drugs in above two studies and there were few studies which compared the responses after using clinically relevant doses of IT ropivacaine and levobupivacaine combined with opioids. Since most IT labor analgesia are conducted through the combination of local anesthetics and opioids, the assessment of analgesic potency of clinically relevant doses of these drugs combined with opioids is of clinical interest.

Consequently, we performed this study to compare the analgesic potency of clinically effective doses of IT ropivacaine and levobupivacaine combined with fentanyl for labor analgesia.

\section{Materials and Methods}

This prospective, randomized, double-blinded study was approved by both the Institutional Review Board of our hospital and the Korean Food and Drug Administration, and written informed consents were obtained from all participating subjects. We recruited 60 American Society of Anesthesiologists physical status (ASA PS) 1 or 2 parturients in early labor (i.e., cervical dilatation $<5 \mathrm{~cm}$ ), with a singleton cephalic presentation at term (> 37 weeks) who requested neuraxial labor analgesia. Parturients with multiple pregnancies, systemic diseases, fetus with known or suspected congenital abnormalities, had visual analog scale (VAS) scores for pain of less than $50 \mathrm{~mm}$, and administration of parenteral or oral analgesics before initiation of neuraxial analgesia were excluded from the study.

Before initiation of analgesia, the following parameters: maternal age, height, weight, gestational age, amount of cervical dilatation, use of oxytocin, parity, and status of membrane were recorded. Baseline pain score was assessed using a VAS (100 $\mathrm{mm} ; 0=$ no pain and $100=$ worst pain imaginable) before the CSE.

The sealed envelope technique was used to randomly allocate all parturients into two groups to receive one of the following: intrathecal ropivacaine (Naropin ${ }^{\circledR}$, Astrazeneca, Södertälje, Sweden) $3 \mathrm{mg}$ in normal saline $2 \mathrm{ml}$ combined with fentanyl 20 $\mu \mathrm{g}$ (group $\mathrm{R}, \mathrm{n}=30$ ) or intrathecal levobupivacaine (Chirocaine, Abott Laboratories, Italy) $3 \mathrm{mg}$ in normal saline $2 \mathrm{~mL}$ combined with fentanyl $20 \mu \mathrm{g}$ (group L, $\mathrm{n}=30$ ). No placebo was used in the study because a deliberate dural puncture performed for this purpose was deemed unjustifiable by the committee.

After establishing venous access and prehydration with 500 $\mathrm{ml}$ lactated Ringer's solution, the combined spinal-epidural analgesia, using a double-segment technique, was performed with the parturient in either the left or right lateral position. The spinal block was performed at the L3-4 or L4-5 interspace under sterile conditions, using a midline approach with a 25 -gauge whitacre spinal needle. After observing a free flow of cerebrospinal fluid (CSF), the spinal study drug was injected intrathecally. Following subarachnoid injection, the spinal needle was withdrawn and the epidural space was identified with a 17-gauge Tuohy needle (Arrow ${ }^{\circledR}$, Arrow International, Philadelphia, USA), using a loss-of-resistance to the air technique at the L2-3 or L3-4 interspace. Next, a 19-gauge epidural catheter (FlexTip Plus ${ }^{\circledR}$, Arrow Inc, Reading, PA, USA) was inserted and secured 3-4 cm into the epidural space, via the Tuohy needle. The epidural catheter was aspirated to confirm the absence of blood or CSF, but epidural test dose was not administered and the parturient was turned into a supine position.

Maternal blood pressure and heart rate were measured, noninvasively, throughout the study period. Maternal hypotension was defined as a systolic arterial pressure $<90 \mathrm{mmHg}$ or $<30 \%$ from the baseline, and was treated by increasing the intravenous infusion rate, and if necessary, administering intravenous ephedrine. The fetal heart rate was also monitored via an exter- 
nal cardiogram throughout the study period. The occurrence of late or variable decelerations or fetal bradycardia of less than 110 beats/min was documented and an obstetrician was consulted when necessary.

In the present study, the primary and secondary outcomes were the duration of analgesia and incidence of complete analgesia, respectively.

Parturients were asked to inform the moment the VAS score decreased below $(10 \mathrm{~mm}$ ) after undergoing CSE procedure. The difference between that moment and intrathecal injection was defined as the onset of analgesia. Also, thirty minutes after the intrathecal injection, the following parameters: pain score by VAS score, the highest sensory level, and degree of motor block were assessed. The highest dermatomal sensory block was tested in each dermatomal level bilaterally for the loss to pinprick sensation. Pinprick response was measured using 25-gauge whitacre spinal needle. Motor block was assessed by using a modified Bromage scale [14] (Table 1), evaluating the ability to raise a leg for 30 seconds, and investigating whether perineal squeezing was preserved or not. Further, duration of analgesia (our primary outcome) defined as the time between the end of the spinal injection and the moment additional analgesia requested by parturients owing to the reappearance of labor pain was assessed. At that time, $7 \mathrm{ml}$ of $0.2 \%$ ropivacaine was administrated via epidural catheter and the patient-controlled epidural analgesia device was connected and started. In addition, parturients showing incomplete analgesia (i.e., VAS $\geq 10$ ) were determined to assess the incidence of complete analgesia (secondary outcome).

Within the first 30 mins after each spinal injection, adverse effects resulting from neuraxial block, such as pruritus, nausea, and vomiting, were recorded and compared in the two groups.

Statistical analyses were performed with SigmaStat software version 3.1 (Systat Software, Inc., Point Richmond, CA, USA). Patient and obstetric data were collected and presented as the mean \pm SD or median (interquartile range), as approciate. Analyses were carried out using the unpaired student's $t$ test and Mann-Whitney U test for comparisons of parametric and nonparametric data between the two groups and with chi-square tests for dichotomous data. The sample size of this study was computed to detect a 30 min difference, with an SD of $40 \mathrm{~min}$ in duration of analgesia between ropivacaine and levobupivacaine based on the

Table 1. Modified Bromage Scale (Intensity of Motor Block)

$1=$ Complete block (unable to move feet or knees)

2 = Almost complete block (able to move feet only)

3 = Partial block (just able to move knees)

$4=$ Detectable weakness of hip flexion (between scores 3 and 5)

5 = No detectable weakness of hip flexion while supine (full flexion of knees)

$6=$ Able to perform partial knee bend results of the previous study $[4,13]$, with a power greater than $80 \%$ and $\alpha=0.05$. In all tests, significance level was set at $\mathrm{P}<0.05$.

\section{Results}

Sixty parturients were enrolled in this study. There were no technical problems with the administration of the intrathecal study drugs or placement of the epidural catheters. All patients commencing the study finished the study period successfully and all were included in the subsequent statistical analysis.

There was no difference in the demographic characteristics or obstetric characteristics between parturients in the ropivacaine and levobupivacaine groups (Table 2). Baseline pain score on a VAS were obtained before CSE and time to VAS minimum after CSE were also similar in two study groups (Table 3 ).

The highest sensory level by pinprick test was higher in the group L than in the group $\mathrm{R}$ [group $\mathrm{R}$ : group $\mathrm{L}=\mathrm{T} 8.5$ (T6-T10) : T6 (T5-T8), $\mathrm{P}<0.05]$. The incidence of VAS greater than 10 was higher in the group $\mathrm{R}$ than in the group $\mathrm{L}$ [group $\mathrm{R}$ : group $\mathrm{L}=8(27 \%): 1$ (3\%), $\mathrm{P}<0.05]$. The duration of analgesia was longer in the group $\mathrm{L}$ than in the group $\mathrm{R}(122 \pm 56 \mathrm{~min}$ vs. $87 \pm$ $41 \mathrm{~min}, \mathrm{P}<0.05)$ (Table 3).

Table 2. Demographic Data

\begin{tabular}{lcc}
\hline & $\begin{array}{c}\text { Group R } \\
(\mathrm{n}=30)\end{array}$ & $\begin{array}{c}\text { Group L } \\
(\mathrm{n}=30)\end{array}$ \\
\hline Age (yr) & $32.2 \pm 3.4$ & $33.4 \pm 4.0$ \\
Weight $(\mathrm{kg})$ & $66.0 \pm 7.2$ & $68.4 \pm 10.6$ \\
Height $(\mathrm{cm})$ & $161.5(158.7-167.2)$ & $164.5(158.0-165.5)$ \\
Gestational age (wk) & $39.5(38.0-40.3)$ & $40.0(39.0-40.6)$ \\
Nulliparous patients (\%) & $22(73)$ & $19(63)$ \\
Cervical diameter (cm) & $3(3-4)$ & $3(3-4)$ \\
Oxytocin use (\%) & $22(73)$ & $25(83)$ \\
Ruptured membrane (\%) & $6(20)$ & $12(40)$
\end{tabular}

Values are mean $\pm S D$, median (interquatile range) or number (\%). No significant differences were found.

Table 3. Analgesia, Sensory \& Motor Block, and Side Effects

\begin{tabular}{lccl}
\hline & $\begin{array}{c}\text { Group R } \\
(\mathrm{n}=30)\end{array}$ & $\begin{array}{c}\text { Group L } \\
(\mathrm{n}=30)\end{array}$ & P value \\
\hline VAS (Pre-block) & $84(75-90)$ & $75(70-80)$ & 0.121 \\
Time to VAS minimum (min) & $6.4 \pm 3.1$ & $5.8 \pm 2.3$ & 0.402 \\
Incidence of VAS $\geq 10$ & $8(27)$ & $1(3)$ & $0.026^{*}$ \\
Duration of analgesia (min) & $87 \pm 41$ & $122 \pm 56$ & $0.022^{*}$ \\
Sensory level & $\mathrm{T} 8.5(\mathrm{~T} 6-\mathrm{T} 10)$ & $\mathrm{T} 6(\mathrm{~T} 5-\mathrm{T} 8)$ & $0.007^{*}$ \\
Leg raising (normal) & $30(100)$ & $29(97)$ & 1.000 \\
Bromage scale (6) & $30(100)$ & $26(87)$ & 0.112 \\
Perineal squeezing (normal) & $23(77)$ & $14(47)$ & $0.007^{*}$ \\
Fetal bradycardia & $2(7)$ & $2(7)$ & 1.000 \\
Pruritus & $23(77)$ & $24(80)$ & 1.000 \\
\hline
\end{tabular}

Values are mean $\pm \mathrm{SD}$, median (interquatile range) or number (\%). VAS: visual analogue score. ${ }^{*} \mathrm{P}$ value $<0.05$. 
None of the patients exhibited motor impairment or perineal squeezing problems before the anesthetic procedure. Thirty mins after the intrathecal injection, the results of leg raising test and modified Bromage scale were not different between the two groups. However, more patients in the group $\mathrm{R}$ preserved the ability of perineal squeezing than in the group L [23 (77\%) vs. 14 (47\%), $\mathrm{P}<0.05$ ] (Table 3).

In addition, the incidences of side effects such as pruritus, nausea, vomiting, and fetal bradycardia resulting from neuraxial block were comparable among the two groups.

\section{Discussion}

Our current trial was designed to clarify the relative analgesic potency of clinically relevant doses of IT ropivacaine and levobupivacaine, combined with fentanyl for labor analgesia. The results of our study demonstrated that $3.0 \mathrm{mg}$ of IT levobupivacaine, mixed with fentanyl $20 \mu \mathrm{g}$, provide a significantly longer duration of analgesia and higher incidence of complete analgesia compared with relevant doses of IT ropivacaine for early labor analgesia. In addition, IT levobupivacaine used for labor analgesia cause a slight weakness in the ability of perineal squeezing.

The comparison of the equivalent doses of IT two local anesthetics, used as a part of CSE technique for labor analgesia, has been reported by previous studies. Lim et al. [4] found that $2.5 \mathrm{mg}$ of IT ropivacaine and levobupivacaine provide a similar duration of analgesia. Furthermore, another study evaluating the MLAD of IT levobupivacaine and ropivacaine described that although levobupivacaine is $20 \%$ more potent than ropivacaine, there were no significant differences in the duration of analgesia between two IT drugs at clinically effective doses of $2.5 \mathrm{mg}$ or greater [13]. On the other hand, we added fentanyl to the two local anesthetics to promote faster onset and more potent analgesia, and our study showed that $3 \mathrm{mg}$ of IT levobupivacaine combined with fentanyl produced a significantly longer duration of analgesia and more incidences of complete block compared with equivalent doses of ropivacaine. These findings are inconsistent with the results of Van de Velde et al. [12] evaluating the relative analgesic potency of IT bupivacaine, levobupivacaine, and ropivacaine, combined with sufentanil for labor analgesia, using full dose-response curves of the three drugs, which described that IT bupivacaine is most potent, and IT levobupivacaine and ropivacaine are similar in the analgesic potency. Although an opioid was added to IT local anesthetics in both studies, there were some differences in the study design and subjects between the two studies. The previous study was designed to investigate the full dose-response relation of the IT local anesthetics; whereas, our study was performed to clarify the analgesic efficacy of the clinical equivalent IT dose of the local anesthetics. In addition, parturients in more advanced labor (i.e., cervical dilatation <
$7 \mathrm{~cm}$ ) were included into the previous study, while our study's subjects were confined to parturients during the early stage of labor. These differences in the study design and subjects might explain a discrepancy in the analgesic potency hierarchy of the local anesthetics between the two studies.

In addition to providing potent pain relief, drugs and their administration methods that induce lesser side effects are required to assure optimal labor analgesia. Motor impairment, known as a most common drawback among cruel side effects relating to labor analgesia, may influence the ability to bear down and relax the pelvic muscles, and can result in abnormal presenting fetal part descent and dystocia [15]. Thus, current obstetric analgesic practice aims to provide effective pain relief, while minimizing motor blockade. In the present study, the degree of motor block was assessed by using a modified Bromage scale, and the extent of motor block estimated by a modified Bromage scale was not significantly different between the IT ropivacaine and levobupivacaine groups. The results of our study are consistent with those of the previous studies, suggesting that there are no difference in the degree of motor block assessed by Bromage score between IT ropivacaine and levobupivacaine at these clinical dose $(2.5 \mathrm{mg}$ or greater) [4,13]. Furthermore, the authors also asked the participants in the present study to rate the ability to squeeze their perineal muscles, and found that squeezing was subjectively impaired in more parturients received levobupivacaine than in those received ropivacaine. The sixteen parturients received IT levobupivacaine showed a significantly weaker perineal squeezing compared with that of the seven who received IT ropivacaine. These findings could be explained by the lower lipid solubility of ropivacaine compared to levobupivacaine augmenting a differential sensory motor block property when administered by IT route [11]. However, it was not assessed that whether the significant weakness in the capacity of perineal squeezing illustrated in parturients who received levobupivacaine was actually associated with decreased maternal satisfaction and increased the risk of instrumental and cesarean delivery or not in our study.

Apart from the duration of analgesia, in practice, the onset time of analgesia would also be one of the crucial considerations in evaluating the suitability of CSE analgesia during early labor. Thus, the authors also investigated the onset time of analgesia, which was defined as time to VAS minimum (VAS $<10 \mathrm{~mm}$ ), and found that there was no significant difference between the two study groups. This finding is in close agreement with the results of one previous study, evaluating the full dose-response relation of IT bupivacaine, levobupivacaine, and ropivacaine, combined with sufentanil for labor analgesia, which demonstrated that IT bupivacaine provided most fast onset of analgesia and IT levobupivacaine and ropivacaine offer comparable onset time of analgesia during labor analgesia [12]. 
According to a previous study comparing the duration of IT labor analgesia between the early and advanced labor stage, the duration of spinal analgesia with sufentanil/bupivacaine was significantly shorter during the advanced labor periods than early labor [14]. These findings were explained by the extent of cervical dilatation and changes of pain input nature as labor analgesia. For these reasons, our study's subjects were confined to parturients during the early stage of labor, and the degree of cervical dilation was not actually different between the ropivacaine and levobupivacaine groups. Further study on the duration of analgesia induced by clinically relevant doses of IT ropivacaine and levobupivacaine with fentanyl during advanced stage of labor is required.

There were several reports, which stated that there is an association between IT opioids and fetal bradycardia during labor analgesia $[15,16]$. Moreover, a recent review article suggested that the combination of opioids and local anesthetics is not recommended during early labor analgesia followed by early initiation of epidural analgesia (within $30 \mathrm{~min}$ after spinal injection) because IT opioids induce fetal heart rate abnormality and the sole IT local anesthetics could promote fast onset and potent analgesia [17]. However, early stage labor pain is primarily visceral in origin; whereas advanced stage labor pain is mainly mediated by somatic nociceptive input [14] and opioids are generally known for offering analgesia by means of treating visceral pain unlike local anesthetics, which primarily reduce somatic pain
[18]. Therefore, the combination of IT opioids and local anesthetics would be recommended during early labor analgesia.

There were several limitations to this study. First, the authors did not compare the analgesic potency of these new local anesthetics to that of IT bupivacaine, which has been used most extensively for CSE labor analgesia. However, several studies have demonstrated that IT bupivacaine used as a part of CSE provides more potent analgesia compared with IT ropivacaine and levobupivacaine during labor analgesia [4,11]. Second, although maternal satisfaction in labor analgesia is paramount considerations, comparisons of parturients' satisfaction scores for neuraxial analgesia between IT ropivacaine and levobupivacaine were not conducted in the present study. Further study on the comparison in maternal satisfaction provided by administration of IT ropivacaine and levobupivacaine during labor analgesia is required.

In conclusion, although both $3 \mathrm{mg}$ of IT levobupivacaine and ropivacaine combined with fentanyl as a part of CSE could be used to provide proper pain relief during early labor, intrathecal levobupivacaine was more effective with respect to analgesic potency. However, IT levobupivacaine produced some motor weakness compared with intrathecal ropivacaine. Therefore, our study proposed IT ropivacaine, owing to its property of less motor block, would be recommended during early labor for preventing prolongation in the second stage of labor caused by labor analgesia.

\section{References}

1. Collis RE, Baxandall ML, Srikantharajah ID, Edge G, Kadim MY, Morgan BM. Combined spinal epidural (CSE) analgesia: technique, management, and outcome of 300 mothers. Int J Obstet Anesth 1994; 3: 75-81.

2. Hughes D, Hill D, Fee JP. Intrathecal ropivacaine or bupivacaine with fentanyl for labour. Br J Anaesth 2001; 87: 733-7.

3. Vercauteren MP, Hans G, De Decker K, Adriaensen HA. Levobupivacaine combined with sufentanil and epinephrine for intrathecal labor analgesia: a comparison with racemic bupivacaine. Anesth Analg 2001; 93: 996-1000.

4. Lim Y, Ocampo CE, Sia AT. A comparison of duration of analgesia of intrathecal $2.5 \mathrm{mg}$ of bupivacaine, ropivacaine, and levobupivacaine in combined spinal epidural analgesia for patients in labor. Anesth Analg 2004; 98: 235-9.

5. Graf BM, Zausig Y, Zink W. Current status and clinical relevance of studies of minimum local-anaesthetic concentration (MLAC). Curr Opin Anaesthesiol 2005; 18: 241-5.

6. Capogna G, Celleno D, Fusco P, Lyons G, Columb M. Relative potencies of bupivacaine and ropivacaine for analgesia in labour. Br J Anaesth 1999; 82: 371-3.

7. Lyons G, Columb M, Wilson RC, Johnson RV. Epidural pain relief in labour: potencies of levobupivacaine and racemic bupivacaine. Br J Anaesth 1998; 81: 899-901.

8. Polley LS, Columb MO, Naughton NN, Wagner DS, van de Ven CJ. Relative analgesic potencies of ropivacaine and bupivacaine for epidural analgesia in labor: implications for therapeutic indexes. Anesthesiology 1999; 90: 944-50.

9. Boulier V, Gomis P, Lautner C, Visseaux H, Palot M, Malinovsky JM. Minimum local analgesic concentrations of ropivacaine and levobupivacaine with sufentanil for epidural analgesia in labour. Int J Obstet Anesth 2009; 18: 226-30.

10. Polley LS, Columb MO, Naughton NN, Wagner DS, van de Ven CJ, Goralski KH. Relative analgesic potencies of levobupivacaine and ropivacaine for epidural analgesia in labor. Anesthesiology 2003; 99: 1354-8.

11. Camorcia M, Capogna G, Columb MO. Minimum local analgesic doses of ropivacaine, levobupivacaine, and bupivacaine for intrathecal labor analgesia. Anesthesiology 2005; 102: 646-50. 
12. Van de Velde M, Dreelinck R, Dubois J, Kumar A, Deprest J, Lewi L, et al. Determination of the full dose-response relation of intrathecal bupivacaine, levobupivacaine, and ropivacaine, combined with sufentanil, for labor analgesia. Anesthesiology 2007; 106: 149-56.

13. Sia AT, Goy RW, Lim Y, Ocampo CE. A comparison of median effective doses of intrathecal levobupivacaine and ropivacaine for labor analgesia. Anesthesiology 2005; 102: 651-6.

14. Viscomi CM, Rathmell JP, Pace NL. Duration of intrathecal labor analgesia: early versus advanced labor. Anesth Analg 1997; 84: 1108-12.

15. Mardirosoff C, Dumont L, Boulvain M, Tramer MR. Fetal bradycardia due to intrathecal opioids for labour analgesia: a systematic review. BJOG 2002; 109: 274-81.

16. Van de Velde M. Combined spinal-epidural analgesia in labor. Anesthesiology 2000; 92: 869-70.

17. Loubert C, Hinova A, Fernando R. Update on modern neuraxial analgesia in labour: a review of the literature of the last 5 years. Anaesthesia 2011; 66: 191-212.

18. Wong CA. Labor analgesia: is there an ideal technique? Anesth Analg 2009; 109: 296-8. 